\title{
COMMENT DÉFINIR LES PRODUITS ALIMENTAIRES?
}

L'élaboration des références pour l'application de la loi du 1er août 1905 entre expertise et consensus professionnel

\section{Pierre-Antoine Dessaux}

Armand Colin | « Histoire, économie \& société »

2006/1 25e année | pages 83 à 108

ISSN 0752-5702

ISBN 9782200921422

DOI 10.3917/hes.061.0083

\section{Article disponible en ligne à l'adresse :}

https://www.cairn.info/revue-histoire-economie-et-societe-2006-1-page-83.htm

Distribution électronique Cairn.info pour Armand Colin.

(C) Armand Colin. Tous droits réservés pour tous pays.

La reproduction ou représentation de cet article, notamment par photocopie, n'est autorisée que dans les limites des conditions générales d'utilisation du site ou, le cas échéant, des conditions générales de la licence souscrite par votre établissement. Toute autre reproduction ou représentation, en tout ou partie, sous quelque forme et de quelque manière que ce soit, est interdite sauf accord préalable et écrit de l'éditeur, en dehors des cas prévus par la législation en vigueur en France. Il est précisé que son stockage dans une base de données est également interdit. 


\title{
Comment définir les produits alimentaires? L'élaboration des références pour l'application de la loi du $1^{\text {er }}$ août 1905 entre expertise et consensus professionnel *
}

\author{
par Pierre-Antoine DESSAUX
}

\section{Résumé}

La loi du $1^{\text {er }}$ août 1905 sur la répression des fraudes et des falsifications marque un tournant dans la police des marchés de denrées et de biens de consommation courante en déléguant à l'administration un pouvoir réglementaire. Pour autant, elle ne se réduit pas à l'avènement d'un pouvoir d'expertise. Les premières années d'application de la loi voient la mise en place de procédures consensuelles de détermination des références pour les transactions. Industriels et commerçants restèrent au cœur des définitions de produits et purent ainsi consolider les structures de marché qui émergeaient à la Belle Époque.

\section{Abstract}

At the beginning of the 20th century, the french "pure food" debate ruled out the american way of standards promoted by Harvey Wiley. The law, adopted on the 1st of august 1905, and still the basis of commercial regulation, gave extended reglementary power to an ad hoc administration but denied the ability of science, and especially chemistry, to enact definitions of goods. These remained a matter of merchants and producers and a process had to be settled to produce global consensus. It involved international conferences that settled, in 1908-1909, the very first codex alimentarius. It also strengthened market structures that were to last for the whole century.

La loi du $1^{\text {er }}$ août 1905 marque, pour la France, un tournant majeur dans la lutte contre les fraudes et les falsifications. Intégrée en 1993 dans l'arsenal européen de protection des consommateurs, elle a constitué la base d'une approche moderne, générale et répressive de ces comportements dolosifs et néfastes au développement commercial. En définissant des principes généraux, elle a consolidé un échafaudage juridique initialement hétéroclite car construit autour de produits spécifiques comme les engrais, le beurre ou encore le vin. La notion de «loyauté commerciale», cœur de

* Une version abrégée de cet article a fait l'objet d'une présentation au colloque AFHE et CHEFF, Pour une histoire de la fraude et de la contrefaçon, Paris, novembre 2004. 
l'évaluation des transactions en cas de litiges, s'en est ainsi trouvée considérablement renforcée. Initialement destinée aux produits alimentaires et pharmaceutiques, cette autre loi de 1905 a enfin rapidement prouvé sa pertinence pour l'encadrement des échanges et son champ a fait l'objet d'un élargissement à l'ensemble des marchandises (décret-loi du 14 juin 1938). La loi de 1905 a donc permis la mise en place de procédures de répression des fraudes suffisamment générales pour être étendues à l'essentiel des transactions. Elle impliquait en effet un véritable service national de répression des fraudes assis sur un pouvoir d'enquête et une faculté d'expertise. Réparti entre les laboratoires des ministère de l'Agriculture et des Finances, il unifia les diverses démarches jusque-là essentiellement menées au niveau municipal et fut un des éléments du renforcement d'un marché national. Enfin et surtout, reconnaissant l'impossibilité d'une mobilisation efficace de l'appareil législatif en ces matières, la loi donnait à l'administration la faculté de statuer en matière de fraudes et de falsifications et de réagir ainsi au mieux à la capacité d'innovation des fraudeurs.

Bien que centrale dans la régulation des marchés de consommation et fondamentale pour la définition de la place de l'autorité publique dans la garantie des transactions, la loi du $1^{\text {er }}$ août 1905 n'a suscité, en histoire économique et sociale, qu'un intérêt limité (même dans le cadre de son centenaire). L'histoire juridique interne de la répression des fraudes la considère comme l'origine d'une intervention publique omnipotente et éclairée en matière de qualité des produits ${ }^{1}$. Les travaux de Nathalie Jas suggèrent de la replacer dans la perspective d'une diffusion dans l'espace social de l'expertise chimique et agronomique et d'y voir une étape importante de la reconnaissance de ce nouveau pouvoir de la science, de la mise en convergence du droit, de l'action administrative et des compétences scientifiques ${ }^{2}$. En forçant probablement un peu trop ce trait, Franck Cochoy et Roland Canu, proposent de la considérer paradoxalement comme un moment clé de la construction d'une politique de la consommation alors qu'elle est clairement centrée sur les relations entre producteurs et commerçants ${ }^{3}$. Enfin, et surtout, envisagée à partir de la question viticole, qui fut effectivement centrale dans son élaboration, la loi du $1^{\text {er }}$ août 1905 peut être comprise comme le propose Alessandro Stanziani comme un moment clé de refondation des relations marchandes

1. Guy-Alexandre Gallon, Le service de la répression des fraudes et du contrôle de la qualité (Ministère de l'Agriculture et du développement rural) des origines à nos jours. 1907-1973, Paris, thèse de droit Université Paris II, 1973 et les deux colloques organisés à l'occasion de son centenaire qui n'ont avant tout réunit que des professionnels et des juristes (Centenaire de la loi du $1^{\text {er }}$ août 1905 sur les fraudes et les falsifications en matière de produits et de services. Cent ans d'action publique, de la loyauté des transactions à la protection du consommateur dans un monde ouvert, Université de Perpignan, septembre 2005 ; Un siècle entre protection du marché et protection du consommateur, Université de Pau, novembre 2005).

2. Évoquant précisément la loi du $1^{\text {er }}$ août 1905, Nathalie Jas explique ainsi comment «L'élaboration des normes de vente et d'analyse est elle aussi normalisée. Elle se déroule, à huis clos, dans des commissions spécialisées, composées de grands savants qui ont tout pouvoir en la matière. Ces commissions constituent ainsi un bon moyen pour imposer les résultats de la recherche» (Nathalie Jas, Au carrefour de la chimie et de l'agriculture. Les sciences agronomiques en France et en Allemagne, 1840-1914, Paris, Éditions des Archives Contemporaines, 2001, p. 321 et plus généralement la première du chapitre 5).

3. Roland Canu, Franck Cochoy, «La loi de 1905 sur la répression des fraudes. Un levier décisif pour l'engagement politique des questions de consommation?», Sciences de la Société, n 62, mai 2004, p. 69-91, suggèrent une interprétation théorique stimulante mais très discutable empiriquement par défaut d'une contextualisation vraiment précise. L'extériorité des consommateurs dans le processus mis en place par la loi, qui les prenait néanmoins pour cible, a par ailleurs été maintes fois signalée. Cf. par exemple, PierreAntoine Dessaux, «Une coopération conflictuelle au cœur de la consommation de masse: les relations industrie-commerce dans l'alimentaire en France, 1900-1970» et Alessandro Stanziani, "Alimentation et santé sous la III République (1870-1914)», dans Alain Chatriot, Marie-Emmanuelle Chessel (dir.), Au nom du consommateur. Consommation et politique en Europe et aux États-Unis au XX siècle, Paris, La Découverte, 2004.

हु) ${ }_{\text {S }}^{\mathrm{H}} 1,2006$ 
dans une époque marquée par l'aggravation des doutes quant aux qualités des produits. D'après son analyse, la crise viticole de la fin du XIX ${ }^{\mathrm{e}}$ siècle ne se limitait pas à des problèmes quantitatifs de production. Ces derniers émergèrent du fait d'un éclatement des normes de production dû à des techniques de vinification. Les relations marchandes s'en trouvèrent fortement déstabilisées ce qui rendit nécessaire un nouvel accord collectif sur la notion même de vin que la loi du $1^{\text {er }}$ août 1905 permit de mettre en œuvre ${ }^{4}$. L'application de la loi du $1^{\text {er }}$ août 1905 a donc conduit, pour les vins, au renouvellement du consensus relatif au fonctionnement du marché et de la concurrence à partir d'une définition des produits et des méthodes d'analyse nécessaires non seulement au bon fonctionnement du service de répression des fraudes mais plus généralement aux transactions courantes. Elle a ainsi contribué à une refondation du marché.

Même si sur les autres marchés la déstabilisation commerciale n'avait pas atteint un tel degré, l'application de la loi sur les fraudes a mis en place des logiques comparables. Là où la pratique libérale des affaires, caractéristique du XIX ${ }^{\mathrm{e}}$ siècle, renvoyait les contentieux commerciaux à une grande liberté d'appréciation des magistrats quant à l'évaluation du contenu des contrats et au respect ou non des accords, la loi du $1^{\text {er }}$ août 1905 substituait la possibilité du recours à des références précises. Ces dernières pouvaient de plus mobiliser d'autres acteurs que les seuls contractants à commencer par les experts scientifiques et leurs méthodes puis, bien que plus tardivement, des hygiénistes ou même des consommateurs. Le cadre des relations marchandes et la construction des marchés du $\mathrm{XX}^{\mathrm{e}}$ siècle s'en trouvait donc modifiés. Les marchés de consommation courante ont ainsi été liés à des règlements d'administration publique. On peut donc s'étonner de ce que les modalités de production de ces règlements aient si peu été étudiées. On peut effectivement s'attendre, comme le suggère Alessandro Stanziani, à ce que le lobbying ait pu y jouer un rôle majeur et que la production des règlements reflète sinon un consensus du moins un rapport de forces suffisant pour leur concéder une certaine légitimité. Le règlement permet alors de faire le constat d'un accord et de désigner les acteurs pertinents d'un marché ou, du moins, reconnus comme tels. L'étude des processus propres à ces constructions a donc beaucoup à apporter à l'histoire de la construction des marchés de la consommation de masse.

$\mathrm{Si}$ une telle étude reste encore largement à faire pour le $\mathrm{XX}^{\mathrm{e}}$ siècle ${ }^{5}$, faute en est sans doute d'abord à l'étroitesse des fonds d'archives aisément consultables. Seul le fonds du ministère du Commerce est disponible sur ce point mais il recouvre essentiellement l'élaboration de la loi ${ }^{6}$. Les laboratoires et services de répression des fraudes des ministères de l'Agriculture et des Finances, pièces maîtresses du dispositif n'ont eux pratiquement rien versé aux archives. À défaut d'une enquête interne qui reste apparemment à mener, il est néanmoins possible d'essayer de reconstituer une partie des modalités d'élaboration des règlements d'administration publique, et tout particulièrement de ceux concernant la définition initiale des produits, à partir de sources imprimées. Les enjeux économiques, juridiques mais aussi professionnels autour des experts-chimistes et du réseau de laboratoires, ont en effet conduit à un foisonnement de publications avant la Première Guerre mondiale. Le recours à des commissions, dont certaines ont publié des comptes rendus d'activité, et surtout à des congrès utilisés

4. Alessandro Stanziani, Histoire de la qualité alimentaire, Paris, Le Seuil, 2005.

5. La recherche sur les commissions de qualité développées après 1945 reste semble-t-il tout autant à faire que celle sur les modalités d'application de la loi du $1^{\text {er }}$ août 1905.

6. Archives Nationales (AN), F12 6970. Il y a des fragments plus tardifs dans les séries contemporaines mais leur ampleur est limitée. 
comme tribunes pour affirmer des orientations, construire et légitimer des définitions de produits a également suscité d'importantes publications. Il est donc possible de proposer ici une première approche de cette question, qu'une recherche antérieure sur la formation d'un marché de produits alimentaires manufacturés, celui des pâtes alimentaires, permet de mettre en perspective ${ }^{7}$. On peut ainsi réévaluer la place effectivement prise par l'expertise scientifique dans ces démarches et mettre en lumière la logique conçue par les maîtres d'œuvre de la loi du $1^{\text {er }}$ août 1905 pour rendre consensuelle la réglementation.

\section{Expertise et définition des marchés}

Loin d'être totalement innovante en matière de répression et de recours à des expertises comme à des règlements d'administration publique, la loi sur les fraudes adoptée le $1^{\text {er }}$ août 1905, mais déposée en 1898 par Jules Méline, est une généralisation de la démarche mise en œuvre dans la loi du 4 février 1888 sur les engrais. Celleci était, de fait, déjà allée extrêmement loin en matière de soumission du marché à une régulation largement définie par des experts relevant du pouvoir administratif.

\section{Une généralisation de la loi sur les engrais}

En 1884, lors de son premier passage au ministère de l'Agriculture, Jules Méline engage une procédure de révision de la loi sur les engrais de 1867 considérée comme particulièrement déficiente. Le ministère estimait en effet qu'en continuant de s'appuyer sur une administration de la preuve postérieure aux actes délictueux et sans disposer de références précises sur la définition des produits, la réglementation du commerce des engrais s'avérait incapable d'enrayer des pratiques commerciales d'autant plus néfastes qu'elles risquaient de détourner le monde agricole de l'effort d'amélioration de la productivité permis par les engrais. Méline commande donc un rapport à un aréopage d'experts scientifiques de haut niveau; parmi lesquels Boussingault, Girard, Grandeau, Pasteur et Schloesing, chargés de dénoncer les limites de la procédure en cours et de proposer des solutions plus efficaces. Rompant avec l'analyse libérale des contrats en partant du constat de la présence d'une forte asymétrie dans la vente d'engrais et, par suite, d'une infériorité de l'agriculteur par rapport au commerçant, ces experts proposent une modification de la logique de répression et d'administration de la preuve ainsi que la construction d'une information claire sur la nature des produits. Ils proposent de faire remonter les délits en amont des transactions effectives, dans l'intentionnalité délictueuse qui peut apparaître dans le stockage de produits falsifiés, d'imposer un étiquetage clair des produits ou, du moins, de factures définissant explicitement l'objet de la transaction, en composition et en origine, et enfin de mobiliser le réseau des stations agronomiques pour procéder à des analyses dont les termes étaient à définir. En dépit d'une opposition résolue des associations industrielles des producteurs d'engrais, qui font valoir la difficulté d'une définition claire de la nature des engrais et des risques de procédures abusives à l'encontre des producteurs et des commerçants, Jules Méline dépose le 14 août 1884 un projet de loi qui prévoit que les règlements d'administration publique à venir définissent les méthodes d'analyse et, implicitement, la composition utile des produits. Ils doivent s'appuyer sur les travaux d'une commission consultative essentiellement composée de chimistes même si elle

7. Pierre-Antoine Dessaux, Des vermicelliers au groupe Danone: consommer, produire et vendre des pâtes alimentaires en France. XVII ${ }^{e}-X X^{e}$ siècles, thèse d'histoire sous la direction de Patrick Fridenson, Paris, EHESS, 2003, à paraître aux éditions de l'EHESS. 
prévoit de donner la parole aux industriels. Ce projet marque donc un net infléchissement de la logique libérale d'analyse de la nature des transactions en reportant la responsabilité sur les vendeurs et la définition in fine des produits hors marché, dans les mains du pouvoir administratif. Il rencontra de sérieuses réticences mais fut finalement adopté en 1888 sur la base de l'accord qui put être trouvé quant à la nécessité de protéger le monde agricole ${ }^{8}$.

Le projet de loi que Jules Méline dépose le 6 avril 1898 devant le Sénat n'est d'abord qu'une extension à l'ensemble des produits alimentaires des principes établis pour la répression des fraudes en matière d'engrais. Avec la crise de la fin du siècle et l'urbanisation qui ont engendré un allongement des circuits d'approvisionnement et le recours à des agents de conservation et une différenciation souvent extrême des qualités de produits effectivement proposés à la vente, la suspicion à l'égard des denrées alimentaires est alors générale. Elle se traduit par de sérieuses craintes en matière d'hygiène publique et mobilise des laboratoires municipaux en plein développement. Des lois spécifiques sont votées pour le vin, le lait, le beurre et la margarine, mais l'idée d'une loi générale apparaît avec un premier projet inabouti en 1895 et relancé en 1898. Assis sur le constat déjà établi quinze ans plus tôt pour les engrais de la nécessité d'une certaine protection des consommateurs et sur l'intérêt d'une procédure répressive capable d'être en phase avec les pratiques des fraudeurs, il propose pour les produits alimentaires une démarche tout à fait similaire. Dans l'esprit de Jules Méline, si la science est apte à désigner clairement les cas de fraude et de falsification au travers d'expertises, elle ne peut devenir un auxiliaire efficace de la justice que si l'on donne là aussi à l'administration les moyens concrets de suivre au plus près les pratiques des fraudeurs. Dans cette perspective, l'éventuelle régulation des marchés ne peut être cantonnée au pouvoir législatif, généralement trop lent par rapport à l'urgence requise par des transactions courantes et potentiellement dolosives, mais doit être concédée à un pouvoir administratif agissant sur une base réglementaire ${ }^{9}$. Reprenant les termes de la loi sur les engrais, le projet prévoit ainsi que des «décrets rendus dans la forme des règlements d'administration publique prescriront les mesures à prendre pour assurer l'exécution de la présente loi» et «indiqueront, sur l'avis du comité consultatif des stations agronomiques et des laboratoires agricoles, la procédure à suivre pour fixer les méthodes d'analyse propres à déterminer les éléments constitutifs, la teneur en principes utiles des marchandises et reconnaître les fraudes» (art. 10) ${ }^{10}$. Les décrets ne sont donc censés permettre que la sélection des méthodes d'analyse mais il ne pouvait échapper aux intérêts concernés que la définition même des éléments à analyser n'est qu'une définition indirecte du produit correspondant. En s'accordant sur une procédure réglementaire, l'administration s'arrogeait implicitement le droit de définir les produits et par suite le cadre des transactions marchandes.

8. Sur la loi du 4 février 1888 et son rôle dans l'institutionnalisation de l'agronomie en France voir Nathalie Jas, Au carrefour de la chimie et de l'agriculture. Les sciences agronomiques en France et en Allemagne, 1840-1914, Paris, Éditions des Archives Contemporaines, 2001, chap. 5, p. 293-325.

9. «Les dispositions du présent projet permettront, à l'avenir, de ne plus recourir à des mesures législatives particulières chaque fois que les progrès de la falsification révéleront la nécessité d'une intervention des pouvoirs publics pour protéger contre la fraude une catégorie de denrées non encore réglementée par une loi spéciale», $J O$, documents parlementaires, Sénat, séance du 6 avril 1898, p. 406.

10. Ibid., p. 409. 


\section{Réticences et résistances}

«Quelle doit être la nature, la qualité substantielle, la composition et la teneur en principes utiles d'un produit alimentaire? Nul ne saurait le dire; non seulement ces définitions n'existent pas, mais elles ne sauraient exister. En effet, il est inadmissible à moins de prétendre réglementer la nature qu'il y ait des marchandises types, des produits alimentaires types, dont la composition soit déterminée d'avance d'une manière officielle et légale. Et si l'on admettait ces types, que deviendrait la concurrence, qui permet au consommateur de s'approvisionner de qualités en rapport avec les prix qu'il veut payer; elle disparaîtrait et avec elle la liberté du commerce». Le jugement porté en 1901 par Gustave de Kester, rapporteur de la commission économique de la Chambre de Commerce et d'Industrie de Paris, sur le projet de loi et instruit par les remarques de quelques chambres syndicales et organisations consulaires, est d'emblée sans appel. «L'ensemble du nouveau projet de loi devrait être purement et simplement rejeté ${ }^{11}$. Une partie du monde professionnel s'insurge donc contre l'idée de types ou de standards de qualité des produits. Ces derniers sont selon eux en contradiction totale avec le principe fondamental de liberté du commerce. Mieux, c'est la capacité même des experts qui est contestée aussi bien dans leurs pratiques que dans leurs prétentions à déborder le cadre de la science pure.

$\mathrm{Si}$ le pouvoir concédé aux experts et aux règlements d'administration publique pouvait être toléré pour le commerce des engrais, d'autant que la loi fut relativement peu appliquée ${ }^{12}$, il n'était nullement question de le voir étendu à l'ensemble des produits touchant à l'alimentation. Contrairement à l'idée que défendait Méline, la stabilisation des résultats scientifiques était connue pour être loin d'être acquise. La chimie avait certes fait d'immenses progrès à la fin du XIX $X^{\mathrm{e}}$ siècle mais l'incertitude concernant la reproductibilité des expériences, la dépendance des analyses de conditions dont tous les paramètres étaient encore loin d'être explorés, était notoire. La confiance des professionnels à l'égard des scientifiques ne pouvait donc être que très relative. Voter la loi c'est donc pour Gustave de Kester, mettre «le vendeur à la merci des conclusions d'un expert qui est cependant toujours faillible» ${ }^{13}$. Pour Gustave Berry, un des adversaires les plus acharnés de la loi lors de sa discussion à l'Assemblée, la loi aurait accordé «le don d'infaillibilité à des chimistes dont les théories sont, tous les jours, combattues par leurs confrères eux-mêmes» et d'en conclure: «laissons l'infaillibilité aux conciles seuls, mes chers collègues ${ }^{14}$. Enfin la démarche scienti-fique dans laquelle les experts les plus en vue, membres pour nombre d'entre eux de la Société chimique de Paris, étaient engagés, semble très loin des préoccupations du commerce tout en étant susceptible, par le biais de la loi, de venir en perturber le fonctionnement. La question de la «chimie pure» engagée autour de la standardisation des procédures d'analyse et de l'unification des nomenclatures, occupe en effet une large part de l'activité des chimistes français dans cette dernière décennie du XIX ${ }^{\mathrm{e}}$ siècle ${ }^{15}$. Il

11. Rapport de Gustave de Kester, séance du 13 février 1901 de la CCIP. Le dossier préparatoire de ce rapport est consultable au service des archives de la CCIP, boîte III 4.42 et contient des remarques formulées par la chambre syndicale des distillateurs en gros de Paris, des vins et spiritueux de Paris, du syndicat de l'alimentation de gros et de la chambre de commerce de Bordeaux. Le rapport est également présent dans le dossier C 5659 de l'Assemblée Nationale déposé aux Archives Nationales mais l'on peut par contre s'étonner du peu de réactions syndicales suscitées par la loi ce qui renvoie à la question de la représentation des intérêts patronaux dans ces domaines (voir infra).

12. Voir Natalie Jas, Au carrefour..., op. cit.

13. Rapport du 13 février 1901.

14. JO, Assemblée Nationale, séance du 10 novembre 1904, p. 2356. 
n'était donc pas infondé de craindre une tentative de généralisation de leurs méthodes hors du champ des éléments chimiques fondamentaux d'autant que les hygiénistes et surtout les discussions états-uniennes sur la réglementation des fraudes poussaient de fait dans le sens d'une définition de «l'aliment pur». Dans cette perspective, la science se serait emparée d'un des fondements de l'activité marchande, la définition des produits.

Les résistances furent suffisamment fortes pour que le projet ne progresse que très lentement et l'ouverture de la discussion, à l'Assemblée, le 10 novembre 1904, fit encore l'objet d'une tentative d'ajournement de Georges Berry. Elle échoua cette foisci devant la volonté gouvernementale, affichée par le ministre de l'Agriculture, Joseph Ruau de mener le projet à son terme. Le contexte de crise viticole, qui motivait la recherche de règles plus claires en matière de définition et d'appellation des vins, lui était également plus favorable. Bien que largement soutenu par l'assemblée qui rejeta l'essentiel des amendements, le projet fait l'objet d'une discussion approfondie qui est l'occasion de préciser clairement ses orientations. Signe d'un large accord initial, les dispositions les plus fondamentales des articles 1 et 11 touchant à la définition de la fraude et des falsifications et au recours aux règlements d'administration publique sont moins discutées que les articles relatifs à la reconnaissance des délits et à leur répression. De fait, les discussions et le vote des amendements expriment clairement le rejet de toute dérive hygiéniste emmenée par Édouard Vaillant et le groupe socialiste. Ce dernier propose en effet de faire «une loi de protection de la santé publique et de l'alimentation publique» à partir d'une «fixation des types normaux des aliments» nommément inspirée de la démarche nord-américaine afin de donner un cadre strict à une «police municipale alimentaire» fondée sur un corps d'inspection ramifié qui aurait eu pour fonction de contrôler l'ensemble des denrées en circulation aussi bien au départ des zones de productions qu'à l'arrivée dans les zones de consommation ${ }^{16}$. Mais, comme le rappelle Léon Mougeot, premier ministre de l'Agriculture amené à intervenir dans la discussion: «nous faisons une loi sur les fraudes et non une loi d'hygiène publique», une ligne fermement tenue par son successeur, Joseph Ruau. Dans cette perspective, il n'est nullement question d'encadrer les transactions dans des définitions ou des prescriptions particulières, mais uniquement de donner un cadre à des échanges loyaux. La loi ne vise nullement à substituer la puissance publique aux acteurs du marché dans la définition du contenu de leurs transactions mais à donner à la répression des fraudes les moyens nécessaires à son application. La fraude est définie comme tromperie sur la nature ou la quantité tandis que la falsification consiste en l'altération des biens dans les deux cas par rapport aux qualités et quantités sousjacentes au contrat.

Si cette lecture précise emporte bien une majorité, il n'en reste pas moins que l'essentiel des transactions s'effectue sur une base coutumière qui n'est jamais systématiquement explicitée. En conséquence, comme le souligne Édouard Vaillant, le

15. Voir Ulriche Fell, «The chemistry profession in France: the Société Chimique de Paris/de France 1870-1914», dans David Knight, Helge Kragh (dir.), The making of the chemist. The social history of chemistry in Europe, 1789-1914, Cambridge, Cambridge University Press, p. 15-38.

16. Édouard Vaillant intervient sur ces points lors de la discussion générale ( $O O$, séance du 17 novembre 1904 , p. 2485-2487) puis lors de la discussion de l'article 11 par dépôt de deux amendements (JO, séance du 23 février 1903, p. 491-494 et p. 496). Le premier amendement proposait la création de ce corps d'inspection tandis que le second proposait «un règlement d'administration publique (qui) établira la définition... et donnera valeur légale à cette définition, de l'état normal ou sain des aliments, denrées alimentaires et tous les autres produits et marchandises visés par la présente loi». 
recours à des définitions de produits est d'une façon ou d'une autre nécessaire de même qu'une véritable définition de l'architecture et des moyens du service de répression des fraudes, questions par lesquelles il espère voir s'affirmer un tournant plus hygiéniste qui lui est justement refusé. Le gouvernement préfère sur ces points s'en tenir à une approche pragmatique: il élude la question de la dépense afférente à cette généralisation des expertises jusque-là essentiellement engagées sur une base municipale ou dans le réseau agronomique et s'en tient à une promesse formelle de recours à toutes les autorités compétentes pour définir les termes des règlements d'administration qui, lors du vote, s'en tiennent aux modalités de mise en vente, aux inscriptions, marques et appellations et modalités d'expertise tout en conservant une éventuelle faculté d'extension ${ }^{17}$. Pour élaborer ces règlements, d'ampleur initialement limitée et essentiellement techniques, Joseph Ruau souligne solennellement en séance prendre «l'engagement dès maintenant de m'entourer de toutes les garanties possibles suivant les indications que le Parlement voudra bien me fournir» et affirme «je suis le ministre de l'Agriculture et ma parole lie non seulement moi-même mais aussi mes successeurs ${ }^{18}$.

Probablement largement adoptée parce qu'elle donnait un cadre général à la protection des appellations viticoles, la loi du $1^{\text {er }}$ août 1905 marque bien une évolution importante dans l'encadrement des marchés mais demeure, lors de son vote, tout à fait libérale. Elle vise en effet essentiellement à donner des moyens d'action à un service à créer dont la tâche est d'abord de procéder à des expertises normalisées dans le cadre défini par les usages commerciaux. L'expertise constitue bien une référence et non un moyen d'intervention. Mais la loi n'en possède pas moins un potentiel d'encadrement important, dont les formes et la légitimité restent pour l'heure à créer.

\section{Mettre la loi en œuvre}

Comme le prévoyait sa logique même, une fois la loi votée, tout restait à faire aussi bien en matière d'organisation d'un service administratif opérationnel que de promulgation des références à mettre en œuvre. Fidèles à l'esprit affiché dans le cadre du débat parlementaire, les responsables de ce nouvel ensemble cherchèrent à limiter autant que faire se peut l'extension de la loi, mais des amendements, tout à fait prévisibles, durent être votés pour que l'ensemble gagne une certaine efficacité. L'extériorité de l'administration à la définition des usages marchands, gage de la légitimité de ses règlements, fut en revanche préservée tandis que la représentation des industriels dut être construite.

17. «Art. 11 - Il sera statué par des règlements d'administration publique rendus, suivant les cas, après avis des comités consultatifs ou techniques compétents, sur les mesures à prendre pour assurer l'exécution de la présente loi, notamment en ce qui concerne: $1^{\circ}$ la vente, la mise en vente, l'exposition et la détention des denrées, boissons, substances et produits qui donneront lieu à l'application de la présente loi; $2^{\circ}$ les inscriptions et marques, indiquant soit la composition, soit l'origine des marchandises, soit les appellations régionales et de crus particuliers, que les acheteurs pourront exiger sur les factures, sur les emballages ou sur les produits eux-mêmes, à titre de garantie de la part des vendeurs, ainsi que les indications extérieures ou apparentes nécessaires pour assurer la loyauté de la vente et de la mise en vente; $3^{\circ}$ Les formalités prescrites pour opérer les prélèvements et procéder contradictoirement aux expertises sur les marchandises suspectes; $4^{\circ}$ Le choix des méthodes d'analyse destinées à établir la composition, les éléments constitutifs et la teneur en principes utiles des produits ou à reconnaître leur falsification; $5^{\circ}$ Les autorités qualifiées pour rechercher et constater les infractions à la présente loi, ainsi que les pouvoirs qui leur seront conférés pour recueillir des éléments d'information auprès de diverses administrations publiques et de concessionnaires de transports» (JO, Chambre des députés, séance du 23 février 1905, p. 491).

18. JO, Chambre des députés, séance du $1^{\text {er }}$ février 1905, p. 494 et 497. 
Conformément aux engagements pris devant la Chambre, Joseph Ruau commença par n'associer à la loi qu'une organisation légère, des commissions chargées de définir les fameux règlements d'administration publique. Par arrêté du 15 novembre 1905 sont donc instituées une commission des boissons, vins et spiritueux et une «commission des corps gras alimentaires, conserves alimentaires, sucres et confiseries, chocolats, matières amylacées, épices, produits coloniaux etc.». Par décret du 15 décembre 1905 est créée une «commission permanente de recherche et de contrôle des procédés d'analyse». Le 3 janvier 1907 enfin apparaît une commission pharmaceutique. Cette décomposition du travail réglementaire ne répond pas seulement à une apparente spécialisation, elle recouvre aussi une urgence inégale selon les secteurs et traduit clairement les motivations profondes du vote de la loi. À chaque commission correspondent en conséquence des logiques particulières.

La commission des boissons, dont les enjeux ont dans une large mesure motivé le vote de la loi, rassemble ses 43 membres désignés et démarre ses activités dès la publication de l'arrêté. Son organisation est relativement claire: les professionnels, négociants, représentants syndicaux et viticulteurs, y sont présents en nombre mais, à 21, ils ne disposent pas de la majorité absolue. Celle-ci échoit en théorie à une coalition potentielle de 17 fonctionnaires, dont d'éminents experts chimistes comme Eugène Roux, du laboratoire municipal de Paris et Fernand Bordas, du laboratoire du ministère des Finances, et de 5 personnalités politiques, dont James Hennessy. Ce dernier, clairement associé aux intérêts viticoles vient toutefois garantir une forme singulière de représentation de «l'intérêt général». La commission se divise ensuite en trois sous-commissions spécialisées. La première, et la plus importante, dite «commerciale» rassemble 28 membres soit quelques fonctionnaires et tous les professionnels. Ces derniers se saisissent dès la première séance de la question de la définition du vin ouverte par la crise viticole et que l'administration aurait préféré voir traitée par la seconde commission, «technique», qui rassemble les chimistes ${ }^{19}$. La dernière «juridique», n'a en fait qu'un rôle secondaire, essentiellement à visée administrative. Incapables de remettre en cause cette saisine de la première commission, les représentants de l'administration sont en fait conduits à l'orienter vers une approche d'intérêt général en suggérant une enquête sur les usages viticoles. Le matériau récolté en 1906 permet ainsi de proposer, sans parvenir encore à un accord définitif et complet, à une définition à la fois suffisamment précise et ouverte pour reconstruire un consensus depuis longtemps défaillant. La notion de définition de produit, qui avait tant agité les bancs de la Chambre et suscité un rejet massif de la notion d'aliment pur, prend ainsi un sens nouveau, essentiellement pragmatique que souligne une des premières thèses de droit consacrée à la loi: «il s'agit avant tout pour cette sous-commission de définir les denrées conformément aux règles de la logique, qui exige qu'une définition s'applique à tout objet défini et ne convienne qu'à lui, et de délimiter exactement les régions de production ${ }^{20}$. Ce premier aboutissement confère une certaine légitimité au cadre juridique ouvert en 1905 dans le monde des producteurs qui se voient ainsi directement engagés dans la construction réglementaire, ce qui répondait de fait à l'intention préventive sous-jacente à cet édifice répressif. Mais l'expérience viticole, fondée sur un

19. Ministère de l'Agriculture, Commission chargée d'élaborer les règlements d'administration publique pour l'application de la loi du $1^{\text {er }}$ août 1905 sur la répression des fraudes, compte rendu des travaux, 19051907, Paris, Imprimerie Nationale, 1908 et pour le débat sur la définition du vin: Alessandro Stanziani, art. cité.

20. Charles Gosset, De la répression des fraudes dans la vente des marchandises: loi du $1^{\text {er }}$ août 1905, Paris, Université de Paris, 1906, p. 138. 
univers professionnel largement mobilisé n'était pas nécessairement reproductible dans les secteurs «etc.» de la seconde commission, appelée à englober le reste des productions alimentaires.

À défaut, sans doute, d'une représentation professionnelle véritablement efficace, la seconde commission présente une physionomie tout à fait différente ${ }^{21}$. Bien que destinée à un nombre nettement plus important de produits, elle ne comprend que 27 membres, dont 19 scientifiques et fonctionnaires, parmi lesquels 13 siègent également dans la commission boissons. Les professionnels sont donc largement minoritaires même s'il s'agit de personnalités importantes comme Gaston Menier, Jules Prevet ou Paul Rouvier ${ }^{22}$. Seules les industries du chocolat, de la laiterie, des graisses et des conserves sont ainsi directement représentées. La meunerie et les industries de transformation des céréales, l'industrie du sucre, de la confiserie et des confitures, la charcuterie et les salaisons, les condiments et les épices ou encore les eaux minérales ne semblent donc pas avoir été en mesure sinon de disposer de représentants du moins de justifier une présence dans cette commission. Bien que l'urgence de la réglementation ait été bien moindre, cette commission ne semble donc pas avoir été en mesure de s'engager dans une procédure similaire à celle des boissons. Les premiers règlements sont effectivement décrétés en 1907 mais sur la base des lois déjà existantes pour les beurres ou pour les huiles et graisses sur la base d'une définition de la pureté et des mélanges tout à fait évidente. Les arrêtés concernant les autres produits n'apparaissent en fait qu'à partir de 1910, après qu'une autre procédure ait été mise en place pour des objectifs élargis.

L'orientation prise par la commission des boissons contredisait clairement les préventions des rapporteurs de la loi du $1^{\text {er }}$ août 1905 comme des élus relativement à l'opportunité de procéder à des définitions de produits. Les viticulteurs en avaient clairement fait le cœur de la réglementation des boissons et, par suite, rien n'empêchait de généraliser cet élargissement des prérogatives réglementaires. On revenait de fait sur l'exclusion de la logique de l'aliment pur car cette dernière pouvait désormais apparaître comme issue d'une logique professionnelle bien plus que scientifique. Le 27 juin 1907, Paul Cazeneuve, qui avait dénoncé en séance les contradictions de la loi votée deux ans auparavant, dépose à l'assemblée en procédure d'urgence une proposition de loi destinée à permettre aux règlements d'administration publique de préciser «la définition et la dénomination des marchandises quelles qu'elles soient, conformément aux usages commerciaux ${ }^{23}$ par amendement de l'article 11. La discussion, qui s'étale jusqu'au vote du 5 août 1908, voit Joseph Ruau émouvoir l'assemblée en soulignant que sa loi ne permet pas de contrer la vente de «vin infâme» au motif que cela ne saurait constituer une fraude. La discussion est par ailleurs l'occasion de préciser

21. Les organisations interprofessionnelles nationales de l'industrie alimentaire sont surtout, avant 1914, l'Union centrale de l'Épicerie en Gros fondée en 1876 qui devient la Chambre syndicale de l'épicerie en gros en 1886 et l'Union des syndicats de l'alimentation de gros de France créée en 1889. Dans les faits ces deux organisations étaient essentiellement parisiennes et ne représentaient que très imparfaitement la myriade d'associations locales.

22. Gaston Menier, député et président du syndicat des chocolatiers, Jules Prevet, sénateur et président du syndicat des conserves alimentaires, Paul Rouvier, sénateur et président du syndicat des laiteries coopératives du Poitou, maire de Surgères. On trouve également dans la commission Marguery, le président du syndicat de l'alimentation parisienne, Lunel, le président du syndicat des producteurs d'huile d'olive, et Vinay, un important fabricant de produits d'épicerie.

23. Exposé des motifs de la proposition déposée le 27 juin 1907, annexe 1102 citée in Xavier de Borssat, Législation et jurisprudence sur les fraudes et falsifications et les appellations d'origine, Paris, $2^{\mathrm{e}}$ éd., 1909. 
encore la procédure et de souligner à quel point les démarches de définition peuvent se comprendre comme légitimées par leur caractère éminemment professionnel. Comme le souligne Henri Ricard, rapporteur du projet au Sénat, la pratique des deux premières années d'application de la loi a montré que le Conseil d'État, qui avalise en dernière instance les décisions réglementaires, peut permettre de lever les dernières réticences du monde professionnel: «la mission de définir les aliments purs serait particulièrement difficile à remplir pour le Conseil d'État s'il ne s'entourait des renseignements demandés soit aux notabilités agricoles ou scientifiques, soit, en un mot, à tous les intéressés». Il n'est désormais plus scandaleux de préciser «la loi de 1905 est improprement nommée. Elle devrait s'appeler la loi de l'aliment pur» ${ }^{24}$, une qualification qui aurait engendré une obstruction trois ans plus tôt mais qui ne renvoie plus à la seule compétence scientifique. C'est qu'entre-temps, une démarche complémentaire de l'activité des commissions et mobilisant une grande partie du monde professionnel a fait évoluer la notion «d'aliment pur». Ces nouvelles garanties expliquent d'ailleurs pour une part l'adoption en à peine plus d'un an de la proposition Cazeneuve.

La reconnaissance légale de l'élargissement du champ d'application des règlements d'administration publique émerge en fait parallèlement à la mise en place d'une démarche de construction collective des définitions pilotée par le service de répression des fraudes que le ministère de l'Agriculture met en place pour appliquer la loi. C'est d'ailleurs à cette imbrication que l'on peut sans doute imputer l'efficacité de l'ensemble de la démarche et la crédibilité que l'administration et ses experts parviennent à se faire reconnaître.

\section{Des laboratoires aux congrès : les débuts du service de la répression des fraudes}

Ripostant à la proposition d'Édouard Vaillant d'établir, à grand frais, un véritable service de police des aliments, la Chambre n'avait pas cru devoir doter l'application de la loi de moyens particuliers. Le recours à des commissions comme l'absence de conception d'un service de répression spécifique, voire même une inspection particulière, correspondent, en 1906, à une volonté de limiter le développement de structures nouvelles ${ }^{25}$. Il faut un an pour juger cette approche intenable et déboucher, par arrêté du 30 janvier 1907 sur la création d'un «service d'inspection des laboratoires et établissements de vente des denrées et produits pharmaceutiques et alimentaires», qui devient, au ministère de l'Agriculture, le service de la répression des fraudes par arrêté du 21 novembre 1907. Sur proposition de Fernand Bordas, chef du laboratoire du ministère des Finances, professeur suppléant au Collège de France, membre du conseil supérieur d'hygiène et des différentes commissions mises en place en 1905, c'est un autre membre des commissions, Eugène Roux (1860-1948) que Joseph Ruau charge de bâtir, pratiquement à partir de rien, le service de répression des fraudes du ministère de l'Agriculture. C'est alors de l'engagement personnel d'Eugène Roux et du réseau de scientifiques républicains du conseil supérieur d'hygiène, conscients de l'enjeu d'une association nouvelle entre monde industriel et expertise scientifique que vont sortir les bases de nouvelles références pour la régulation des marchés. Dans son hommage nécrologique de 1948, Henri Queuille, expert de l'heure pour les questions alimentaires, n'hésite ainsi pas à en faire celui qui «réussit à construire une doctrine française en matière de répression des fraudes, à la faire admettre dans la pratique,

24. JO, Assemblée Nationale, séance du 20 mars 1908 pour Joseph Ruau et Sénat, séance du 7 juillet 1908 pour Henri Ricard cité dans Xavier de Borssat, Législation..., Paris, 2 éd., 1909, p. 220.

25. Charles Gosset, De la répression..., op. cit., p. 138. 
dans les mœurs, complétant ainsi l'œuvre du législateur ${ }^{26}$. Pour cela il fallait, comme le souligne Maxime Toubeau, un des premiers collaborateurs du service: «un homme qui fût, à la fois, un savant (de préférence un chimiste), un organisateur audacieux et un administrateur prudent, susceptible d'acquérir la confiance de la production et du commerce, capable de concilier des intérêts économiques opposés ${ }^{27}$, un traducteur.

\section{La marque d'Eugène Roux}

Lorsqu'il est chargé, en 1906, d'une mission d'inspection des laboratoires qui pourraient être agréés et mis en réseaux pour assurer l'application de la loi, Eugène Roux a déjà une solide carrière scientifique derrière lui. Parisien, ingénieur agronome et chimiste d'abord intéressé par les engrais, il travaille au laboratoire municipal de Paris où il rencontre Fernand Bordas, puis part au Muséum où il assiste Léon Maquenne et poursuit une recherche sur les dérivés du sucre soutenue en 1903. Dreyfusard militant, il participe également à l'ascension politique de François Deloncle et dispose ainsi de relations politiques solides. D'abord chargé d'évaluer les laboratoires agronomiques et municipaux, il conçoit de créer un inspectorat spécifique et négocie avec Clemenceau le rattachement à l'Agriculture des moyens du service d'inspection des pharmacies du ministère de l'Intérieur. Le service de la répression des fraudes gagne ainsi des locaux au 42, rue de Bourgogne. Une fois passés les décrets et arrêtés nécessaires pour l'inspection, trois collaborateurs sont engagés en 1907: Maxime Toubeau, chargé des affaires juridiques, Henri Fouquet et Octave Valentini. D'abord modestes, les crédits bénéficient rapidement d'une augmentation conséquente liée à la volonté parlementaire de manifester ainsi l'engagement public dans la crise viticole. Cinq nouveaux collaborateurs sont engagés en 1908 dont Albert Bruno, nommé inspecteur général des laboratoires de l'agriculture avec 14 inspecteurs sous ses ordres et trois laborantines qui traduisent là aussi la féminisation précoce de la profession de chimiste. Cette dernière intéresse d'ailleurs directement le développement du nouveau service. Avec la mise en place d'un inspectorat et la définition de méthodes d'analyses unifiées, l'expertise chimique, depuis longtemps reconnue par les tribunaux, doit gagner en professionnalisme ce qui la conduit à se détacher du monde de la chimie académique pour renforcer sa crédibilité dans le monde industriel.

Pour unifier la pratique professionnelle des experts associés à l'application de la loi de 1905, une revue mensuelle voit son premier numéro paraître en novembre 1908. Les Annales des falsifications et de l'expertise chimique, publiées conjointement avec le Bulletin international de la répression des fraudes qui poursuit à Paris, une publication initiée à Amsterdam en 1887 par la commission internationale scientifique et populaire des falsifications des denrées alimentaires. Il s'agit alors clairement d'une publication française qui marque également, comme nous le verrons plus bas, un déplacement à Paris du centre de gravité de la réflexion internationale sur la lutte contre les fraudes. Dirigées par Fernand Bordas et Eugène Roux, les Annales mettent en réseau l'essentiel des acteurs engagés sur le terrain dans la répression des fraudes. Le secrétariat de rédaction est tenu par le chimiste principal du ministère des Finances. Sous le haut patronage des principaux enseignants universitaires de la chimie et de l'hygiène, dont Armand Gautier, le comité de rédaction associe le réseau des inten-

26. Henri Queuille, «In memoriam Eugène Roux», Annales de la falsification et de l'expertise chimique, avril-juin 1948.

27. Maxime Toubeau, «Hommage à Eugène Roux», Annales de la falsification et de l'expertise chimique, janvier 1949. 
dants militaires, alors très actif dans la redéfinition de l'alimentation efficace ${ }^{28}$, et les responsables des principaux laboratoires d'expertise: Calvet des Finances, Gros du laboratoire municipal de Clermont, Lajoux de Reims, Martel de l'inspection vétérinaire, Müntz, de l'INA, Ogier de la Préfecture de Police de Paris, Roux de Poitiers, Schribaux du laboratoire des semences ${ }^{29}$. Ces deux publications, livrées conjointement, mêlent rapports d'expertise et réflexions sur les méthodes d'analyse, bibliographie, analyses des acteurs de la répression des fraudes et textes réglementaires français mais aussi étrangers. C'est bien un véritable travail d'institutionnalisation qui s'y joue et qui débouche logiquement sur la création, en 1912, d'une société des experts-chimistes de France, présidée par Paul Cazeneuve et organisée en six sections qui reprennent les prérogatives de la répression des fraudes: chimie industrielle, alimentation, pharmacie, toxicologie, législation, douanes et contributions, et dont les Annales deviennent l'organe officiel. Parallèlement, Paul Cazeneuve parvient à faire reconnaître officiellement un diplôme d'expert chimiste, gage de l'engagement durable d'une profession dans la lutte contre les fraudes.

La formation des experts ne constitue cependant qu'une fraction de l'activité nécessaire à l'institutionnalisation du service de répression des fraudes. Une activité d'information et d'interprétation juridique apparaît d'autant plus nécessaire que la grande liberté initialement laissée aux règlements d'administration et la progression du travail réglementaire supposent un suivi constant. Les Annales y contribuent directement au travers des publications juridiques de Maxime Toubeau. De nombreuses thèses de droit visent également à faire le point entre 1906 et 1910 sur l'évolution de l'interprétation de cet ensemble de mesures, chronologie qui souligne une certaine stabilisation des questions d'interprétation de la loi à partir de $1911^{30}$, mais c'est surtout un réseau constitué par un petit nombre de juristes, qui vivent directement de l'application de la loi, qui permet d'assurer la diffusion de références communes. En collaboration directe avec Eugène Roux, le procureur de la République Ferdinand Monier et le juge d'instruction Fernand Chesney, rédigent en 1909 un Traité théorique et pratique sur les fraudes et les falsifications qui constitue la référence de l'avant-guerre pour la magistrature et reprend une large part des discussions menées dans les Annales et le Bulletin dont ils sont à la fois collaborateurs et membres du comité de rédaction ${ }^{31}$. Parallèlement, des avocats se spécialisent dans le contentieux lié à l'application de la loi et contribuent à en définir les contours. Paul Bogelot, Albert Crinon et surtout Xavier de Borssat (1870-1942), également collaborateur des Annales, s'imposent comme des médiateurs obligés. Avocat au barreau de Paris, Xavier de Borssat publie ainsi dès 1906 un recueil commenté de la loi, de ses règlements et de la jurisprudence, qui prend une forme de juris-classeur dans l'édition de 1909 et est mis à jour en 1923. Cette publication est largement diffusée par souscription auprès des syndicats profes-

28. Voir Pierre-Antoine Dessaux, Des vermicelliers..., op. cit., p. 101 et suiv.

29. Sur le réseau formé par ces ingénieurs agronomes, voir Nathalie Jas, Au carrefour..., op. cit.

30. Charles Gosset, De la répression..., op. cit., 1906; André Chauvin, Répression de la fraude en matière de vins (jusque et y compris la loi du $1^{\text {er }}$ août 1905, étude de cette loi), Paris, 1908; René Vincent, De la Répression des fraudes et falsifications de denrées alimentaires, loi du ler août 1905, thèse pour le doctorat, Caen, 1909; G. Pagès, Les Falsifications des denrées alimentaires et la loi du $1^{\text {er }}$ août 1905, Montpellier, Montane et Sicardi, 1909; Émile Lansier, Observations critiques sur la loi du $1^{\text {er }}$ août 1905, Poitiers, 1910; S. Krainik, L'évolution de la répression des fraudes et falsifications alimentaires, thèse de droit Paris, Paris, Sirey, 1910 et René Barreyre, La Loi du $1^{\text {er }}$ août 1905 sur les fraudes et le commerce des spiritueux, Paris, A. Rousseau, 1913.

31. Ferdinand Monier, Fernand Chesney, Eugène Roux, Traité théorique et pratique sur les fraudes et les falsifications, Paris, Larose et Tenin, 1909, 2 vol. 
sionnels et fait par la suite l'objet d'une actualisation permanente dans le cadre de la Revue des fraudes, produits purs et d'origine qu'il lance en octobre 1924 et qui paraît jusqu'en $1947^{32}$.

Comme le souligne, bien plus tard, Maxime Toubeau, qui devint par la suite le responsable du service de la répression des fraudes, en 1911: «la partie était déjà gagnée ${ }^{33}$. Le service a alors les moyens de son institutionnalisation et est en passe d'être véritablement opérationnel, de disposer d'une expertise reconnue et acceptée. Mais il ne s'agit là que d'une dimension d'une démarche qui se veut avant tout œuvre de prévention en bâtissant un consensus professionnel sur la notion de fraude et c'est sur ce point sans doute que l'activité d'Eugène Roux est le plus efficace et produit la légitimité dont bénéficie la réglementation et sa mise en œuvre. Confronté à la nécessité immédiate de références pour rendre la loi opérationnelle et donc crédible, mais aussi porteur d'une philosophie de sa mise en œuvre parfaitement cohérente avec les promesses de Joseph Ruau de bâtir sur le consensus et non uniquement sur l'expertise, Eugène Roux assoit à partir de 1908 l'application de la loi sur une démarche à la fois collective et capable, sous réserve d'une organisation idoine, de résultats rapides: les congrès internationaux.

Convaincu que «les lois ne sont bonnes que lorsque leur application rencontre le consentement de ceux-là mêmes qui les subissent; mieux encore, lorsque ces derniers ont travaillé à leur élaboration», ce qui relève de «la vraie méthode républicaine», Eugène Roux, ne défend aucune prétention à la domination de l'expertise en matière de fraude, mais la conçoit plutôt comme en retrait, au service d'une régulation dont les acteurs concernés ont à définir le cadre et voit sa position comme à l'interface entre les pratiques professionnelles de deux mondes, celui de l'expertise fondée sur l'analyse scientifique et celui de la production et du commerce ${ }^{34}$. Il s'agit en fait de conquérir auprès du monde professionnel une légitimité qui est a priori loin d'être acquise. Celle-ci passe d'abord par des prises de position à commencer par le rejet de toute prétention à un déterminisme fondé sur la supériorité des arguments scientifiques ${ }^{35}$. Le premier directeur de la répression des fraudes affiche son rôle comme orienté vers la construction «d'une entente cordiale entre l'Administration, chargée de veiller également sur tous les intérêts, et les représentants du commerce honnête groupés dans leurs syndicats» et voit dans ce processus commun de recherche de l'intérêt général une réponse à «une aspiration générale de notre démocratie» ${ }^{36}$. Aux accusations de corporatisme qui ne manquent pas de surgir, en raison de la possibilité ainsi donnée aux groupes professionnels de définir leur cadre d'activité ${ }^{37}$, Eugène Roux oppose une philosophie de la répression qui repose sur le contrôle ex post des produits que permet

32. Xavier de Borssat, Législation et jurisprudence sur les fraudes et falsifications et les appellations d'origine, Paris, 1906, 1909, 1923. Voir l'hommage que dresse son successeur à la Revue des fraudes, Albert Bonn, dans le $\mathrm{n}^{\circ} 18$ de 1947 de cette revue.

33. Maxime Toubeau, «Hommage à Eugène Roux», art. cité.

34. Eugène Roux, Rapport technique au Premier Congrès de la Croix Blanche, dans Société Universelle de la Croix-Blanche de Genève, Compte rendu des travaux du $1^{\text {er }}$ Congrès International pour la Répression des Fraudes alimentaires et pharmaceutiques, Genève 8-12 septembre 1908, Genève, 1909, p. 208.

35. Eugène Roux conteste ainsi l'idée selon lui d'inspiration marxiste, d'une convergence juridique internationale en matière de législation des fraudes sous la pression de la science et de l'économie défendue par Krainik (S. Krainik, L'évolution..., op. cit., 1910, Eugène Roux, «L'évolution de la répression des fraudes», Bulletin..., 28, février 1911, p. 48).

36. Préface de Xavier de Borssat, Législation..., op. cit., Paris, $2^{\mathrm{e}}$ édition, 1909, p. 9.

37. Il s'agit d'une interprétation libérale tout particulièrement défendue par Henri Berthélemy et Joseph Lemercier, Manuel élémentaire pour la répression des fraudes, Paris, Marchal et Billard, 1909. 
la décomposition chimique et se distingue ainsi de la dimension préventive qu'il voit dans le contrôle corporatif ex ante sur les conditions de production. Dans le cadre répressif «on peut, pour la plupart des produits, laisser la liberté du fabricant s'exercer à sa guise, certain que l'on est de pouvoir, ultérieurement, contrôler l'usage de cette liberté, reconstituer après coup l'œuvre de fabrication, demander des comptes à l'artisan malhonnête» ${ }^{38}$. L'expertise demeure donc en retrait, comme une ressource mobilisable et non comme un cadre initial. Il prévoit d'ailleurs assez rapidement d'y associer les producteurs en soulignant l'importance des analyses organoleptiques dont la maîtrise ne peut être assurée que par des professionnels. Eugène Roux travaille donc à afficher la convergence de ses services avec les intérêts professionnels et en produit la preuve la plus notable dans la procédure qu'il met en place pour établir les définitions et qui ne peut, pour suivre la ligne ainsi affichée, que se détacher de la commission d'experts initialement chargée d'encadrer la production réglementaire pour tous les produits alimentaires autres que les boissons.

Avec le débat, puis le vote, de l'amendement Cazeneuve, le processus réglementaire affiche clairement rechercher à définir des aliments purs. L'esprit du tout nouveau service de répression des fraudes incline à mettre l'administration et l'expertise scientifique en retrait pour conquérir une position légitime auprès des producteurs. Il devient nécessaire de concevoir une procédure capable de susciter une représentation des intérêts du commerce et de la production et de dépasser les divergences locales pour produire des définitions de base des transactions cependant conformes à l'intérêt général. Les hommes de la répression des fraudes vont alors se saisir du mouvement de réflexion engagé à l'échelle européenne par les hygiénistes depuis la fin du XIX siècle pour créer une institution nouvelle, la Croix Blanche de Genève, apparemment internationale, mais qui fut avant tout le laboratoire des définitions de produits nécessaires à la réglementation des fraudes.

\section{La Croix Blanche de Genève}

La nécessité d'une convergence réglementaire au niveau international pour assurer une véritable lutte contre les fraudes dans un univers déjà très mondialisé avait clairement été évoquée, dès la fin du XIX ${ }^{\mathrm{e}}$ siècle dans le cadre des congrès internationaux de médecine et d'hygiène. Un premier vœu en ce sens avait été formulé au congrès médical d'Amsterdam en 1879. En 1882, au congrès d'hygiène et de démographie, Georges Brouardel propose que soient rédigés des rapports sur les législations nationales en vue d'une présentation au congrès d'hygiène prévu en 1884. On en resta cependant au stade des vœux et seule la démarche fut précisée. C'est lors de l'exposition universelle de Bruxelles de 1888 que l'idée d'un codex alimentaire, calquée sur le codex pharmaceutique, serait apparue. Trois ans plus tôt on s'était entendu, également à Bruxelles, pour définir un programme fondé sur la définition des falsifications, l'établissement de procédures d'analyses communes et la recherche d'une convergence des législations nationales. Ces vœux sont encore réitérés à Vienne en 1897 et à Berlin en 1903 mais l'absence d'un véritable soutien étatique, sans doute nécessaire autant en matière de moyens que de crédibilité de ce type de démarche relativement aux enjeux de régulation du commerce international, brida largement ces démarches ${ }^{39}$. Avec la consolidation de réglementations nationales de lutte contre les fraudes autour de 1905 et les

38. Eugène Roux, «La répression des fraudes hier et aujourd'hui», Bulletin..., février 1913, p. 49.

39. S. Krainik, L'évolution... op. cit., Paris, Sirey, 1910, 6e partie. 
besoins propres à la réglementation française, le projet connaît une nette accé-lération: en septembre 1907 apparaît une nouvelle association internationale privée, la Croix Blanche de Genève, dont une des premières ambitions est de promouvoir la convergence réglementaire.

S'inscrivant dans la lignée des associations de lutte contre la tuberculose, la Croix Blanche, domiciliée 43, rue Taitbout à Paris, se donne pour mission d' «accomplir dans la vie civile la tâche que remplit avec tant d'éclat la Croix Rouge sur les champs de bataille» ${ }^{40}$. À cette fin, l'alimentation constitue son objectif central à commencer par la lutte contre les fraudes et les falsifications pour «arriver à assurer à chacun une nourriture saine et reconstituante, procurer à tous "l'aliment loyal" ${ }^{41}$. Placée sous le patronage de Henri de Rothschild, le grand hygiéniste «soutien» de l'œuvre de la Goutte de lait, la Croix Blanche se présente comme une démarche privée, à vocation internationale dont l'initiative revient à François Deloncle qui en est un des fondateurs aux côtés de Jules Harmand et de Raphaël Blanchard, le directeur de l'Institut de médecine coloniale, du Dr. Bouchard de la faculté de médecine de Paris et G. Lachenmayer du Crédit Suisse. Elle rassemble cependant le monde de l'expertise chimique et c'est dans son cadre que sont publiés, à partir de 1908, les Annales et le Bulletin international. L'appui du gouvernement français y joue de fait un rôle crucial, aussi bien en matière de subventions, d'abord ministérielles puis parlementaires, qui couvrent plus des deux tiers du budget de fonctionnement, que par la mise à disposition des experts publics pour ses travaux dans lesquels interviennent Joseph Ruau, Eugène Roux et Fernand Bordas, qui préside la délégation française ${ }^{42}$. La démarche ne s'en présente pas moins comme internationale et non gouvernementale. L'intérêt en est double: officieuse, ses avis ne lient en rien les autorités, s'affichant comme internationale, elle vise à situer les intérêts locaux dans une perspective favorisant la convergence nationale. Elle s'affiche donc volontairement comme orientée vers l'objectif impartial de convergence réglementaire internationale, et place en tête de ses préoccupations «la conclusion d'accords internationaux pour la répression des fraudes et falsifications en ce qui concerne les boissons, les denrées alimentaires et les produits agricoles». À cette fin des congrès sont dès l'origine prévus et annoncés, en septembre 1907 au congrès d'hygiène et de démographie de Berlin par François Deloncle qui se charge de constituer un réseau international avec deux associés. Charles Vuille (1856-1920) avocat du barreau Genève et bâtonnier de l'ordre, député au Grand Conseil et membre du Conseil d'administration du Journal de Genève, préside l'association et assure un ancrage international fondé sur un axe franco-suisse. Paul Bolo, très probablement le Bolo Pacha fusillé le 17 avril 1918 pour intelligence avec l'ennemi, aventurier mondain, proche de Joseph Caillaux, époux d'une riche veuve de négociant de vins de Bordeaux, président de la confédération générale viticole et représentant des vins de

40. AN F12 6970, Courrier de présentation, 1908. Ce dossier rend compte du suivi de la démarche par le Ministère du Commerce. Les travaux des trois premiers congrès de l'organisation ont fait l'objet d'une publication: Société universelle de la Croix Blanche de Genève, Compte rendu des travaux du premier congrès international pour la répression des fraudes alimentaires et pharmaceutiques, Genève, s.e., 1908; Compte rendu des travaux du second congrès international pour la répression des fraudes alimentaires et pharmaceutiques, Paris, s.e., 1909; Compte rendu des travaux du troisième congrès international pour la répression des fraudes alimentaires et pharmaceutiques, Berne, s.e., 1911.

41. Discours d'ouverture du Congrès de 1908 par le président Charles Vuille, Compte rendu..., op. cit., 1908.

42. Joseph Ruau et Joseph Caillaux font voter une subvention exceptionnelle de 40000 francs en juin 1909 au nom de «l'intérêt supérieur du commerce français». Pour 1907 et 1908 le montant des fonds publics n'est pas connu, mais ils sont déclarés «conséquents» lors du premier congrès. 
champagne que ses affaires du tournant du siècle avaient conduit à tisser des liens depuis la Méditerranée jusqu'en Amérique Latine, assure le reste des liaisons internationales ${ }^{43}$. Les réseaux d'affaires et des intérêts coloniaux sont donc mobilisés pour monter rapidement une série de congrès internationaux sous le patronage de scientifiques à la fois experts chimistes et médecins comme le docteur Eugène Richard, médecin principal de l'armée et membre du Comité consultatif d'hygiène publique, et d'Auguste Reverdin, fameux médecin hélvétique, qui assurent tous deux, probablement d'assez loin ${ }^{44}$, la vice-présidence. La position de retrait occupée par les experts scientifiques, pourtant jusque-là moteurs dans les congrès d'hygiène, se retrouve dans l'organisation même des congrès dont le premier se tient dès septembre 1908 à Genève, mobilise 34 délégations officielles ainsi que plus de 900 membres participants, dont deux tiers de français. Six cent se déplacent effectivement pour les travaux et sont, pour l'essentiel, des producteurs. La Croix Blanche parvient donc à s'assurer une large adhésion et donc une certaine crédibilité dans le monde industriel.

Si les publications de la Croix Blanche touchent essentiellement le monde de l'expertise chimique, ses congrès mobilisent au contraire d'abord le monde industriel, en particulier en France et dans l'esprit de la loi du $1^{\text {er }}$ août 1905. Le premier congrès, prévu en 1908 à Genève, se donne pour objectif, «la définition étant à la base de toute résolution», de «définir les Aliments Purs» étant entendu que cette démarche «ne peut réussir que par la collaboration des trois compétences d'ordre distinct intéressées à la question, c'est-à-dire: les Commerçants, les Légistes et les Chimistes. Eux seuls, en effet, peuvent dans une consultation commune, définir L'ALIMENT PUR» ${ }^{45}$. Cette consultation commune n'est cependant pas conçue comme devant se dérouler simultanément, confronter des points de vue dont les logiques sont fondamentalement différentes. Comme l'explique Philippe Dunant, qui préside le premier Congrès de 1908, la démarche se veut progressive et donne la main aux premiers intéressés, les industriels, tandis que les experts ne sont fondés à intervenir que sur la base des orientations que les premiers auront défini:

On s'adressera d'abord aux commerçants et aux industriels. C'est à eux, qui sont aux prises tous les jours avec les questions pratiques du marché des denrées alimentaires et avec les exigences de la clientèle, qu'on demandera tout d'abord ce qu'il faut entendre, dans chaque catégorie de produits par l'aliment pur, et nous parlons ici de l'aliment commercialement pur, c'est-à-dire tel que l'exige et le conçoit l'acheteur qui demande le produit. Nous n'entendons pas demander aux commerçants la définition de l'aliment chimiquement pur (...). Nous demanderons aussi au commerce et à l'industrie de spécifier les manipulations qui doivent être considérées comme licites ou nécessaires. [Alors] un second congrès convoquera les hygiénistes et leur soumettra les résultats de ce premier travail. Les hygiénistes adopteront ou repousseront telles ou telles manipulations demandées, suivant qu'ils les jugeront admissibles ou non, au point de vue spécial de l'hygiène (...). Dans un troisième congrès [les] chimistes auront à établir les formules reconnaissables des différents produits et enfin à unifier les méthodes d'analyse (...). Les juristes interviendront ensuite pour fixer les règles de droit et pour coordonner dans des textes appropriés la matière ainsi élaborée. Enfin par la collaboration finale des diplomates, une entente internationale fixera la base d'une union alimentaire, comme on a codifié internationalement le droit des auteurs et des inventeurs. Nous savons d'ailleurs que l'élaboration d'une convention internationale sera un stimulant puissant pour engager les États à édicter, dans leurs territoires respectifs, des

43. Serge Demidoff, «Un aventurier de grands chemins: Bolo Pacha», Historama, n 27, 1975, p. 73-79; Roger Colombani, Des escrocs magnifiques. Les grands destins, Paris, Flammarion, 1994.

44. Âgé de 59 ans en 1907, Auguste Reverdin décède en 1908.

45. Plaquette de présentation du Congrès, AN F 126970. 
législations sur les fraudes alimentaires. Cette action du droit international sur le droit interne a déjà eu, dans d'autres domaines, les effets les plus bienfaisants ${ }^{46}$.

La recherche de l'aliment pur dans le cadre de la Croix Blanche s'inscrit donc totalement dans la logique impulsée par l'application de la loi du $1^{\text {er }}$ août 1905 . Son organisation n'est d'ailleurs pas sans rappeler celle des commissions instituées en 1905 mais il s'agit de construire des définitions par et pour les producteurs et de ne faire intervenir l'évaluation scientifique qu'au titre d'expertise ex post, ce que traduit une claire succession des tâches. Au niveau international, cela conduit à rompre très clairement avec la démarche plus scientifique qui avait pu prévaloir jusque-là, en particulier aux États-Unis sous la direction de Harvey Wiley. Comme le souligne alors Fernand Bordas, la démarche d'inspiration française s'oppose fondamentalement à l'idée de standard ou de moyenne, dont l'évaluation reviendrait en dernière analyse aux chimistes, dans le droit fil du concept d'élément chimiquement pur, totalement évincé lors des discussions législatives: "Il a fallu renoncer également au système déplorable des moyennes de composition chimique, des standards et autres procédés empiriques qui, dans la pratique, n'ont eu pour résultat que de légaliser en quelque sorte la falsification». La composition des délégations traduit d'ailleurs cette opposition de principe et les différences nationales dans la construction de la législation des fraudes. Les participants français mais aussi allemands, belges, espagnols, portugais et suisses sont essentiellement issus du monde de la production, avec il est vrai des motivations très marquées pour certains produits d'exportation ${ }^{47}$. L'Argentine et le Chili, la Grande-Bretagne, la Hollande, l'Italie, les Pays-Bas et la Serbie mais aussi et surtout les États-Unis, pays plutôt importateurs de denrées alimentaires, ou plutôt relativement moins spécialisés dans des exportations de ce type, présentent des délégations essentiellement composées de savants, hygiénistes, médecins et chimistes. Entre les deux congrès de 1908 et de 1909, le chargé d'affaires de l'Ambassade de France à Washington s'inquiète d'ailleurs de l'impact que pourrait avoir sur ces travaux «la tendance qui s'accentue de plus en plus en ce pays, d'étendre les pouvoirs du bureau de Chimie, [qui] mérite d'être prise en considération... pour se défendre contre les excès de pareilles mesures ${ }^{48}$. De fait si l'affaire est entendue en France, ce sont bien deux approches très différentes qui entrent en compétition au niveau international et sont susceptibles d'avoir des conséquences majeures sur les flux commerciaux. Pour autant, la logique même de la prise de décision dans le cadre des congrès de la Croix Blanche, qui sont alors la seule démarche crédible menée en ce sens au niveau international, et qui se fondent à la fois sur l'adhésion volontaire et sur des votes ad hominem, en assemblée plénière, sur la base des rapports de commissions spécialisées, donne un avantage majeur aux industriels français et contribue sans doute à expliquer leur adhésion au projet. C'est donc d'une logique fondamentalement française, issue des problèmes d'application de la loi du $1^{\text {er }}$ août 1905 , qu'est issue la première ébauche de codex alimentarius.

«Jusqu'ici les pouvoirs publics avaient toujours confié à des administrateurs et à des hygiénistes le soin d'édicter des règlements contre la fraude et les falsifications, sans avoir une connaissance suffisante des usages et des nécessités du commerce que

46. Discours d'ouverture du Congrès de 1908 par le président Philippe Dunant, Compte rendu..., op. cit., 1908, p. 32.

47. On retrouve sans surprise les grands noms de l'industrie du chocolat suisse, des vins ibériques et les représentants des principaux syndicats professionnels allemands.

48. AN F12 6970, la citation de Fernand Bordas est extraite d'une coupure de presse non datée mais qui évoque les congrès. 
ces règlements ont parfois méconnus» rappelle Eugène Roux dans son bilan du premier congrès dont l'esprit consiste au contraire selon lui «au lieu de tenir tous les commerçants pour suspects, et de concerter, en dehors d'eux, des règlements répressifs, c'est avec leur concours, en faisant appel à leur collaboration, qu'il y a lieu de jeter les bases d'une organisation de défense contre les fraudes» ${ }^{49}$. À cette fin, les délégations nationales ont pour mission de mobiliser le monde professionnel pour établir les rapports qui font l'objet des discussions en commission lors des congrès. Pour la France, ce sont 37 syndicats et 14 chambres ou unions syndicales qui participent à la fois au plan collectif et individuel ${ }^{50}$. Les chambres de commerce, également sollicitées, ne sont que 13 à répondre à l'appel. Celle de Beauvais vote une motion de participation au motif qu' «Il est donc utile que les producteurs, industriels et commerçants français s'intéressent au Congrès de Genève. Il est indispensable qu'ils y fassent entendre leur voix, afin de résoudre cette question de l'“aliment pur" dans un sens pratique et utile, et non théoriquement, comme cela arrive trop souvent quand ces questions sont seulement traitées par des savants et des fonctionnaires». Mais celle de Paris reste volontairement en retrait, comme elle l'avait déjà fait pour la commission des boissons, soulignant son incompétence en des matières jugées concerner essentiellement le monde agricole et très probablement au motif d'une opposition durable de Gustave de Kester à l'ensemble de la démarche ${ }^{51}$. Le potentiel de divergence à l'intérieur du monde professionnel appelle par ailleurs une gestion spécifique lorsque l'enjeu international suscité par le congrès ne suffit pas à provoquer les transactions nécessaires au consensus. Fernand Bordas, grand organisateur des rapports français est donc appelé à susciter la conciliation: «dans la pratique, les différents Syndicats d'une même industrie n'ont pas toujours des opinions identiques sur tous les points qui intéressent leur corporation. Il arrive quelquefois que des divergences de minime importance prennent tout à coup une forme aiguë dans les discussions (...) C'est pourquoi nous avons dû pratiquer la politique d'apaisement, et demander quelquefois à un Délégué-rapporteur indépendant, de s'efforcer de concilier les exigences des parties en cause, et de ramener le calme dans les esprits par trop enclins à s'échauffer. Ce procédé a donné d'excellents résultats ${ }^{52}$. Et des rapports sont déposés pour discussion dans le cadre des quatorze sessions spécialisées du premier congrès de septembre 1908.

Le congrès de Genève de 1908 s'appuie donc sur une large mobilisation d'un monde patronal français pourtant encore relativement disséminé. La participation étrangère, bien que loin d'être anecdotique, ne mobilise quant à elle qu'un nombre très restreint d'industriels. Fernand Bordas veut y voir l'impact d'une moindre organisation de la représentation patronale. «Malgré le dévouement de nos Comités, nous ne pouvions espérer réaliser par les mêmes moyens, un résultat analogue et cela pour la raison très simple que, à de rares exceptions près, on ne trouve pas à l'Étranger le rouage indispensable qui nous avait si bien aidé, c'est-à-dire la puissance syndicale» ${ }^{53}$. De fait, les

49. Rapport technique de clôture de Eugène Roux in Société Universelle de la Croix-Blanche de Genève, Compte rendu des travaux du $1^{\text {er }}$ Congrès International pour la Répression des Fraudes alimentaires et pharmaceutiques, Genève 8-12 septembre 1908, Genève, imp. I. Soullier, 1909.

50. Sont ainsi présents des représentants de l'épicerie française, de la boulangerie, confiserie, chicorée, conserve, droguerie, moutarde, salaisons, du commerce de gros des vins cidres et spiritueux, qui inscrit 34 participants, des marchands de beurre de Quimper, des brasseurs et crémiers de Paris, épiciers et négociants d'alcool de Lyon, huiliers de Nice, négociants en beurre, ou encore des mandataires des Halles.

51. Archives CCIP III 4.42.

52. Allocution de Fernand Bordas en ouverture du second congrès.

53. Idem. 
différences de philosophie de la répression des fraudes, la mise en place du premier congrès en moins d'un an, les capacités de mobilisation propres aux organisateurs, et surtout sans doute le jugement porté sur une démarche qui peut apparaître essentiellement franco-française aussi bien dans l'esprit que dans la volonté de promouvoir des définitions et des labels essentiellement favorables aux exportations françaises, ont pu conduire les délégations étrangères à opter pour une position d'observation plus que de partie prenante et à ne cautionner que de loin la démarche. Il n'en reste pas moins qu'elle semble avoir gagné en crédibilité entre le premier et le second congrès d'octobre 1909 à Paris. La présence étrangère, qui se réduit alors à 24 pays, gagne cependant quelque peu en surface au sein du doublement du nombre de participants inscrits. Mieux, les rapports étrangers, initialement très minoritaires sont alors 17 sur les 75 déposés à la discussion. Ils visent essentiellement à défendre des produits locaux comme le whisky, le cheshire ou le paprika, dans l'esprit de nombreuses discussion sur les appellations d'origine qui se déploient sur les congrès et s'inscrivent dans la définition des fraudes, et expriment par là une volonté industrielle de protection qui dépasse largement les frontières françaises, ou se concentrent sur des enjeux importants du commerce international comme avec la définition du chocolat qui oppose déjà suisses et anglais.

L'initiative de la Croix Blanche possède au moins le mérite d'exister et se conçoit de toute façon comme une expérience appelée à devenir une référence. Dans des univers juridiques où la loyauté des transactions peut être appuyée sur des définitions coutumières, une telle démarche est mobilisable au motif qu'elle propose au moins un cadre établi. Dès 1909, Philippe Vuille souligne ainsi que «à plusieurs reprises, en effet, les Tribunaux dans différents pays, en France particulièrement, mais également en Hollande et en Italie, ont pris en considération les résolutions du Congrès de Genève et adopté dans la solution de litiges, les définitions que nous avions admises» ${ }^{54}$. À défaut de règlements nationaux, l'exercice du droit peut valoriser ces références malgré l'absence de conventions internationales et concède aux définitions une valeur stratégique. La procédure de stabilisation des définitions revêt donc une importance particulière. Fondée sur les propositions issues des rapports préalables, elle prévoit une discussion en séances spécialisées dans le cadre des congrès. Celles-ci sont ouvertes à tous les membres intéressés et présidées par les experts scientifiques censés n'avoir dans un premier temps qu'une position d'observateurs. Une proposition de définition est alors établie et soumise en assemblée plénière qui procède au vote qui devrait aboutir, dans le cadre du premier congrès à définir à la fois les produits et les manipulations jugées acceptables pour que les rassemblements ultérieurs soumettent ces décisions des producteurs aux remarques des chimistes et hygiénistes mais sans que ces derniers aient à bouleverser les choix initiaux. Dans les faits, le premier congrès n'aboutit qu'à des propositions de définitions préliminaires et le congrès de Paris de 1909 se trouve de nouveau focalisé sur les définitions et les manipulations. Dans le cadre de cette seconde réunion, les définitions initialement admises sont bien considérées comme intouchables et ne peuvent être que complétées. Les amendements ou objections sont collectés mais non intégrés car le but demeure avant tout de provoquer un consensus en forçant la recherche d'une base commune. La définition doit par ailleurs être suffisamment simple et correspondre à «l'aliment commercialement pur tel que l'exige et le conçoit l'acheteur qui demande le produit» ${ }^{55}$. Pour atteindre cet

54. Allocation de Philippe Vuille en ouverture du second congrès, p. 38.

55. «Compte rendu du Congrès de Genève donné par E. Sohier au congrès international de l'alimentation de Gand», dans AN F12 6970. 
objectif, un avantage majeur était cependant concédé aux intervenants du premier congrès, pour l'essentiel des industriels français parmi les mieux organisés, qui purent ainsi faire prévaloir, comme le montre le cas des pâtes alimentaires, la hiérarchie des produits correspondant le mieux à leurs stratégies.

\section{Qu'est-ce qu'une pâte?}

Si le commerce de pâtes alimentaires ne traverse pas une crise similaire à celle du vin et des alcools, il n'en reste pas moins qu'en ce début de $\mathrm{XX}^{\mathrm{e}}$ siècle, la confiance accordée aux produits s'est écornée aussi bien du fait de pratiques de différenciation des produits aboutissant parfois à des falsifications que de la méfiance plus générale qui accompagne la dépendance croissante de la population envers les produits alimentaires manufacturés. En préambule du dépôt de son projet de loi à l'Assemblée Nationale, Jules Méline est ainsi amené à citer les pâtes diététiques, enrichies en gluten, parmi les produits pour lesquels fraudes et falsifications tendent à devenir courants. L'activité des laboratoires municipaux traduit également cette méfiance au tournant du siècle à travers l'augmentation du nombre d'analyses de produits de type pâtes. De fait, avec le retour à une phase de croissance des prix du blé, la tentation est grande pour certains fabricants soit de proposer des produits de qualité inférieure soit de tricher sur les qualités pour disposer d'une offre compétitive. Comme s'en émeut, en 1893, Jean Carret, principal producteur de haut-de-gamme français, devant ses confrères de la Chambre de Commerce de Lyon: «la production et la vente ont diminué, surtout en ce qui concerne les produits de bonne qualité. La concurrence s'efforçant de produire des pâtes à bon marché, les acheteurs se laissent malheureusement trop aller dans cette voie qui est contraire au développement de la consommation et aux intérêts des acheteurs ${ }^{56}$. De fait, à la question de la fraude et des falsifications s'ajoute celle, plus générale, de la référence des produits écoulés sur le marché. Depuis le milieu du $\mathrm{XIX}^{\mathrm{e}}$ siècle en effet, les producteurs lyonnais, qui se délocalisent ensuite à Marseille, ont opté pour une production à base de blés durs nécessairement importés, et donc plus onéreux mais reconnus comme de meilleure qualité aussi bien sur le plan diététique que culinaire. Cette approche du produit les a d'ailleurs conduit à structurer l'espace des qualités du marché au détriment des autres producteurs et à obtenir une position extrêmement forte sur les marchés d'exportation, en particulier aux États-Unis où la qualité française apparaît un temps mieux reconnue que l'italienne, aussi bien pour des motifs de qualité des productions que des préjugés liés aux immigrants. Autour de la question de la définition des produits nécessaire à l'application de la loi du $1^{\text {er }}$ août 1905, c'est donc la hiérarchie du marché, aussi bien intérieur qu'international, qui est en jeu et avec elle, à terme, la permanence de capacités de valorisation des produits par délimitation de l'espace de la concurrence ce qui explique la forte mobilisation des industriels dans le processus de la Croix Blanche.

D'après les comptes rendus des congrès, l'industrie des pâtes alimentaires y est représentée, au niveau professionnel, par le Syndicat Général des Fabricants de Pâtes Alimentaires de France qui rassemblait des industriels issus de l'ensemble du territoire mais de dimension plutôt importante et par ses homologues suisse et allemand (Verband deutscher Teigwaren-Fabrikanten) ${ }^{57}$. La délégation italienne, au total restreinte à

56. Comptes rendus de la Chambre de Commerce et d'Industrie de Lyon, Lyon, 1893.

57. Compte rendu..., op. cit., 1908 et 1909. Ces deux volumes sont cependant très différents: les comptes rendus du Congrès de Genève (1908) proposent une synthèse plutôt consensuelle des débats, tandis que ceux du Congrès de Paris (1909), nettement plus volumineux (1496p.), rapportent l'intégralité des rapports et des débats. 
une trentaine de participants, ne comprenait de fait aucun industriel de la branche et n'intervient pas dans le débat, peut-être pour ne pas légitimer une procédure lancée par ses principaux concurrents. À cette représentation collective s'ajoutent des membres importants du syndicat: son président, Ferrand, de Lyon, ainsi que Georges Blanc, de Marseille, Courtine, de Maison-Alfort, et Vincent, de Sainte-Colombe. Rivoire et Carret et Bertrand, de Lyon, également membres de l'organisation, disposent de voix utilisables par procuration. En conséquence, les industriels de l'axe Lyon-Marseille, qui avaient renouvelé le marché dans la seconde moitié du XIX ${ }^{\mathrm{e}}$ siècle, ont une majorité relative au sein de l'ensemble français et peuvent s'appuyer sur les semouliers présents comme eux dans la commission des produits farineux. Dès l'ouverture des travaux du congrès de 1908, ils se sentent suffisamment en force pour poser la question de la restriction de la dénomination "pâtes alimentaires» aux seuls produits du blé dur, démarche qui rencontre l'assentiment de leurs confrères helvétiques mais se heurte à la représentation de l'ensemble des intérêts nationaux, pour la France, que rappellent les experts non professionnels ainsi qu'aux pratiques industrielles des autres nations. Une définition aussi restrictive ne pouvant conduire à un consensus, une autre stratégie est donc préférée.

Plutôt que de heurter de front les intérêts de leurs concurrents en s'attaquant à la nature de leur production, les partisans des produits issus des semoules de blé dur cherchent à maîtriser la hiérarchie des qualités légitimes de façon à mettre en avant leur production. Ils obtiennent la restriction de la dénomination «pâtes alimentaires» aux seuls produits du blé, ce qui impose déjà la mention explicite d'éventuels mélanges, permet d'obtenir une définition simple du produit pur et dévalorise de fait toute autre production. Ils obtiennent également l'exclusion des colorants dans les pâtes de qualité courante ainsi que dans les pâtes aux œufs au motif du risque de tromperie qu'un tel emploi peut entraîner. Est ainsi obtenue, de façon détournée, une valorisation des produits du blé dur qui, naturellement plus jaunes que les pâtes ordinaires de froment, blanches ou grises, deviennent clairement identifiables pour le consommateur. Acquise en 1908, cette position n'est, dans la logique du congrès, plus amendable par la suite quand bien même les producteurs suisses défendent en 1909 un retour au statu quo ante suite à l'observation d'un recul de leurs ventes du fait avec de cette définition à laquelle ils avaient pourtant initialement accordé leurs suffrages.

Le succès des producteurs lyonnais se renforce dans le cadre des discussions de 1909. Dans son rapport, Ferrand, cherche à adjoindre à la définition générale la précision selon laquelle les «matières premières sont surtout des produits de mouture des blés durs, riches en gluten, ou quelquefois aussi de blés tendres auxquels on rajoute du gluten, ce qui équivaut parfois à l'emploi de blé dur pur ${ }^{58}$. Cet alinéa ouvre la voie à des analyses de laboratoire plus précises car fondées sur un dosage du gluten. Il est rejeté mais Ferrand réussit à marquer un dernier point. Arguant de l'intérêt de ne pas avoir à justifier de l'origine des matières premières au prétexte qu'elle aurait été impossible à prouver du fait de la division du travail dans la filière, il réussit à faire adjoindre à la définition initiale que «les dénominations Taganrok et Afrique en usage dans l'industrie des pâtes alimentaires pour les macaronis, vermicelles et autres pâtes doivent être considérées comme des désignations de qualités ou d'espèces et non comme des indications d'origine des blés employés à la fabrication des produits ${ }^{59}$. Ces deux dénominations sont censées correspondre aux principales qualités de blé dur

58. Rapport Ferrand, Compte rendu..., op. cit., 1909, p. 243.

59. Ibid., p. 244. 
travaillées à Marseille, ceux d'Odessa et ceux d'Algérie, mais sont effectivement devenues des termes génériques pour désigner les produits de qualité supérieure issus uniquement de blés durs, la qualité lyonnaise. En les mêlant dans une même convention officielle, les industriels concernés voient finalement validée la pratique qui consiste à mélanger ces deux matières premières de qualité et de prix sensiblement différents, ce qui revenait à reconnaître une forme générique de blé dur. En procédant de la sorte ils bénéficient du même coup d'un effet d'appellation d'origine fondée en espèce et non en terroir, une perte pour les représentants russes, qui protestèrent mais ne disposaient pas d'un poids suffisant pour contrer cette proposition en assemblée plénière. Celle-ci adopta donc la définition suivante, appelée à demeurer pour plusieurs décennies la référence du secteur:

On entend par PÂTES ALIMENTAIRES, sans autre qualificatif, des pâtes non fermentées obtenues par pétrissage de semoules ou de farines de froment, avec de l'eau, sans addition d'aucun colorant.

Les pâtes alimentaires obtenues avec d'autres semoules ou farines doivent en porter l'indication.

Sous le nom de pâtes aux œufs, on entend les pâtes alimentaires dans la fabrication desquelles on fait intervenir au minimum 3 œufs par kilo et une coloration reconnue inoffensive par l'usage et qui devra être déclarée.

Les dénominations Taganrok et Afrique indiquent la qualité des produits et non l'origine des blés employés à leur fabrication. Mais ces dénominations ne s'appliquent qu'à des semoules ou des farines de blé dur ${ }^{60}$.

Destinée à produire une convention directement tirée des pratiques des acteurs économiques plutôt qu'à instaurer une norme inspirée par des préoccupations d'intérêt général, la procédure d'établissement des définitions de produits assurée dans le cadre des congrès de la Croix Blanche reflète sans surprise l'état d'un rapport de force entre les intérêts des producteurs. Cette définition des pâtes alimentaires ne recoupe de surcroît nullement les analyses des médecins et des hygiénistes et ne peut se prêter à une modification conforme à leurs appréciations. En effet, les autorités scientifiques ne voyaient aucune raison de défendre une qualité plutôt qu'une autre dès lors que les produits disposaient d'une bonne teneur en gluten éventuellement fondée sur un ajout dans le cadre de la production. Marcel Labbé, une des sommités françaises en matière de régime alimentaire avant-guerre, recommandait pour sa part des pâtes faites de «farine de froment, du beurre et du lait, additionnées ou non d'œufs» ${ }^{61}$. Edmond Jacquet et Guido Rovesi, qui consacrent un article à ce produit dans le premier volume de 1911 du Bulletin de la Société Scientifique d'Hygiène Alimentaire et d'Alimentation naturelle de l'Homme, abondent en ce sens en soulignant que «en un mot, les pâtes bon marché sont plus nourrissantes que les pâtes chères» du fait de l'ajout de gluten ou de céréales plus variées ${ }^{62}$. Les préoccupations scientifiques apparaissent donc totalement décalées par rapport aux intérêts industriels mais ne peuvent, du fait même de

60. Compte rendu..., op. cit., 1909.

61. Marcel Labbé, Régimes alimentaires, Paris, Baillières, 1910, p. 105.

62. Voir chap. 1. Ces deux auteurs soulignaient en effet que «la variabilité dans la qualité des pâtes tient beaucoup moins à l'origine des semoules qu'aux procédés de fabrication et à la proportion de semoule et de farine de blé tendre des mélanges préparés pour l'impastation. Les pâtes sont d'autant plus savoureuses qu'elles contiennent davantage de semoule. Malheureusement le blé dur est plus cher (...). La loi française sur les fraudes alimentaires, si salutaire dans tant de cas, pourrait intervenir là aussi de façon efficace». Edmond Jacquet, Guido Rovesi, «Le renchérissement de la vie et la consommation des pâtes alimentaires», Bulletin de la Société Scientifique d'Hygiène Alimentaire et d'Alimentation naturelle de l'Homme, 1911, vol. 1, p. 358-370. 
la procédure choisie, s'imposer. Pour autant, la définition de 1909 ne peut être réduite à la seule expression d'un groupe d'intérêt qui imposerait ses vues. Plus subtilement, la procédure retenue conduit à cartographier et à hiérarchiser le marché ce qui correspond à la vision d'une différenciation de la production orientée vers la valorisation du produit. Dans ce cadre, c'est la maîtrise des critères de différenciation qui constitue la clé de la définition de niches et de rentes de position ce qui conduit bien à rejeter toute norme ou standard qui créerait une structure plus concurrentielle. Conforme à l'esprit de la loi du $1^{\text {er }}$ août 1905 qui n'a nulle intention de transformer les marchés mais uniquement de leur redonner de la transparence, la définition conventionnelle ainsi négociée structure de fait durablement l'activité de la branche. La référence au blé dur tire vers le haut la qualité moyenne des productions et sera avalisée comme norme lorsque, dans les années 1930, les intérêts des producteurs rencontreront ceux de la politique céréalière et de la préférence impériale ${ }^{63}$. Les productions renforcée en gluten, donc non pures au regard de la définition sont alors contraintes de se situer sur un autre marché, celui des produits diététiques, nettement plus restreint, alors que cette technique de production, pour partie dérivée de la fabrication d'amidon, aurait pu conduire au développement de fabrications à très bon marché. Parce qu'elle repose sur un consensus des producteurs dominants, la définition établie en 1908-1909 contribue donc à définir de façon durable la physionomie du marché des pâtes alimentaires français et par suite sa position relative dans la compétition internationale.

\section{L'impact des congrès}

L'ambitieux projet exposé en ouverture du congrès de 1908 n'alla pas à son terme. Après une remobilisation sans suite par Henri Queuille dans le cadre de la SDN en 1937, il faut attendre le projet de codex alimentarius lancé par la FAO en 1963 pour commencer à voir aboutir, mais dans un tout autre esprit, une procédure de production de conventions internationales en matière de produits alimentaires ${ }^{64}$. Après une phase d'activité internationale, également marquée par sa participation aux congrès internationaux d'interdiction de la saccharine de 1908 et 1909 ainsi qu'aux congrès d'unification des méthodes d'analyse, la Croix Blanche semble avoir disparu parallèlement à la création de la société française des experts chimistes de 1912. Les congrès d'hygiène, de chimie, de droit et diplomatique n'eurent donc pas lieu. Peut-on pour autant en déduire que cette construction visait essentiellement l'application de la loi du $1^{\text {er }}$ août 1905 ?

La production, en deux ans, d'un dictionnaire des principaux produits alimentaires fondé sur les pratiques industrielles signe incontestablement le succès d'une démarche qui aurait pu s'avérer nettement plus longue et délicate dans un contexte purement national. De fait, les décrets d'application, plus directement soumis aux pressions des différents groupes d'intérêts, s'étalent sur plusieurs décennies. Si les orientations prônées dans le cadre de la Croix Blanche ne furent pas nécessairement avalisées dans les règlements ultérieurs, la démarche joua cependant un rôle probablement important dans la construction d'une crédibilité de la volonté de l'administration de faire une loi pour et non contre la production et contribua à légitimer ses décisions. Comme le soulignait Joseph Ruau en ouverture du congrès de 1908, celui-ci devait être compris

63. La loi du 3 juillet 1934 est la première à définir la nature des pâtes alimentaires et rend obligatoire l'emploi de semoule de blé dur.

64. Xavier de Borssat, «De l'opportunité de la répression internationale des fraudes», État Moderne, octobre 1937, p. 220-224; http://www.codexalimentarius.net. 
comme: «le gage définitif de la confiance réciproque qui règne, et qui doit régner, entre eux et les services administratifs de la répression des fraudes» car fondé sur le rappel que: «seuls les commerçants ont autorité pour dire: voilà ce que nous appelons du vin, du lait, du beurre, du chocolat» ${ }^{65}$. Pour autant ce gage ne semble avoir été que très ponctuel, les commissions de réglementation n'ayant apparemment pas fait pas la suite l'objet d'un élargissement au monde industriel et l'administration ayant conservé une large autonomie dans ses décisions réglementaires. Mais les références définies par les industriels étaient là et constituaient désormais le fondement de toute discussion.

Si l'on en croit les ouvrages publiés dans l'entourage du service de la répression des fraudes, les définitions établies dans le cadre des congrès eurent en effet une valeur juridique lorsqu'aucun règlement n'était clairement établi. Deux arguments jouaient en ce sens. D'une part, le service de répression des fraudes s'était largement engagé dans la réalisation des congrès et Eugène Roux comme Fernand Bordas n’avaient pas ménagé leurs efforts pour que les discussions aboutissent à des transactions acceptables. Ainsi, comme le souligne dès 1909, Xavier de Borssat dans son ouvrage de référence: «la tâche de préparer les définitions, dénominations et d'examiner les manipulations permises avait été officieusement assumée par le Congrès international pour la répression des fraudes (...) Il est bien entendu que ces définitions ne lient en aucune façon le Gouvernement français pas plus que le Gouvernement d'aucun autre État, mais la part éminente prise à ce Congrès par le directeur du service de la répression des fraudes est garante du compte qui sera tenu des définitions arrêtées à Genève». Un jugement largement repris dans les thèses et manuels de droit ${ }^{66}$. Un article de 1911 du Bulletin souligne également que «les décisions servent aujourd'hui de base aux réglementations définitives» si bien qu'il convient de reconnaître qu'il n'y a «dans celles-ci par conséquent rien d'arbitraire, ni d'oppressif, puisque les intéressés eux-mêmes ont été appelés à participer à leur élaboration » ${ }^{67}$.

$\mathrm{Au}$ niveau juridique, d'autre part, la nécessité, pour les magistrats, de se référer aux définitions coutumières en l'absence de réglementation précise fait des définitions de la Croix Blanche une référence aisée et largement diffusée dans les manuels de répression des fraudes à commencer par celui publié par Monier, Chesney et Roux: «1210 - Les définitions adoptées par ce Congrès méritent d'être retenues comme étant l'expression des usages commerciaux, puisqu'elles ont été rédigées par les collectivités commerciales les plus importantes de notre pays» ${ }^{68}$. Xavier de Borssat estime quant à lui qu' «en l'absence de règlement d'administration publique, c'est à l'usage commercial qu'il faut se référer, et les usages commerciaux ont été, en quelque sorte codifiés dans les travaux des Congrès de Genève et de Paris » ${ }^{69}$. Il n'hésite pas, en conséquence, à rappeler systématiquement la définition des congrès pour les produits dont la réglementation est pendante. Largement confortée par ces analyses, la jurisprudence avalise donc, le cas échéant, les conventions établies dans le cadre de la Croix Blanche, dans le respect cependant de la jurisprudence déjà établie ${ }^{70}$. Les congrès ont donc permis de

65. Congrès de 1908.

66. Xavier de Borssat, Législation..., 1909, p. 190-191; Émile Lansier, Observations op. cit., thèse de droit, Poitiers, 1910, p. 8.

67. Curtel, «Les fraudes sur les produits alimentaires: leur répression», Bulletin..., février 1911, p. 51.

68. Monier, Chesney, Roux, Traité théorique... op. cit., 1909, p. 510.

69. Xavier de Borssat, Législation..., 1923, p. 55.

70. Voir le recueil de jurisprudence établi dans Xavier de Borssat, Législation... ainsi que Maxime Toubeau, Fraudes et falsifications. Une lutte d'un demi-siècle, Paris, Berger-Levraut, 1957. 
rendre la loi immédiatement opérationnelle tout en assurant la légitimité des décisions juridiques et administratives mais sans lier pour autant l'administration. On peut donc y voir un élément essentiel et particulièrement subtil de l'application de la loi du $1^{\text {er }}$ août 1905.

La mobilisation d'un cadre international, rapidement délaissé après 1910, semble bien n'avoir eu qu'un fondement essentiellement politique. Pour autant, la valeur juridique interne de la démarche était potentiellement transposable dans des système juridique similaires, c'est pourquoi les définitions du congrès de 1909 continuèrent à faire référence bien au-delà des années 1900 . Si cette dimension, considérable, demeure à explorer, elle peut avoir conduit, le cas échéant, à structurer durablement les échanges internationaux de produits alimentaires sur la base de logiques initialement spécifiquement françaises, fondées sur la reconnaissance et la protection de différenciations qualitatives pour partie fondées sur des appellations et des garanties d'origine. Dans cette perspective, l'influence française dans la construction du marché du vin, largement garantie au XX $\mathrm{X}^{\mathrm{e}}$ siècle, par les modalités d'application de la loi du $1^{\text {er }}$ août 1905 , pourrait également se retrouver, sur les mêmes bases, pour nombre de produits alimentaires déjà largement exportés et dotés d'une solide réputation avant la Première Guerre mondiale.

CRH-EHESS / IDHE

\section{Principaux textes d'application de la loi du $1^{\text {er }}$ aô̂t 1908}

* arrêté du 15 novembre 1905: commissions des boissons et autres produits

* arrêté du 15 décembre 1905: commission permanente des techniques d'analyse

* décret du 31 juillet 1906: méthodes de prélèvements et laboratoires agréés

* arrêté du $1^{\text {er }}$ août 1906

* décret du 29 août 1907 et loi du 16 avril 1896: beurre et margarine

* décret du 11 mars 1907: graisses et huiles

*décret du 3 septembre 1907: vin

* décret du 28 juillet 1908: cidre et poirés

* loi du 5 août 1908: précisions à l'art. 11 (déposée le 27 juin 1907)

* circulaire du 20 juillet 1909: absinthe

* décret du 19 décembre 1910: sucre, confiserie, chocolaterie

* circulaire $\mathrm{n}^{\circ} 16$ ministère agriculture (1910): définitions

* décret du 15 avril 1912: charcuterie

* décret du 15 avril 1912: emballages et inscriptions

* circulaires du 3 août 1912 et du $1^{\text {er }}$ novembre 1912: interdiction des additifs chimiques pour la conservation et diverses précisions.

* décret du 15 avril 1915: conserves de fruits et légumes, de poisson 